\title{
Optical tracking of relaxation dynamics in semi-dilute hydroxypropylcellulose solutions as a precise phase transition probe
}

Hernan Garate ${ }^{* a}$, King-Wo Li ${ }^{b}$, Denis Bouyer ${ }^{b}$ and Patrick Guenoun ${ }^{* a}$

${ }^{a}$ LIONS, NIMBE, CEA, CNRS, Université Paris-Saclay, CEA-Saclay, 91191 CEDEX Gif-surYvette, France.*E-mail: hernan.garate@cea.fr (H.G.), patrick.guenoun@cea.fr (P.G.)

${ }^{b}$ IEM (Institut Européen des Membranes), UMR5635 (CNRS-ENSCM-UM), Université de Montpellier, Place E. Bataillon, F-34095 Montpellier, France

\section{Abstract}

Phase separation of thermo-responsive polymers in solution is a complex process, whose understanding is essential to screen and design materials with diverse technological applications. Here we report on a method based on dynamic light scattering (DLS) experiments to investigate the phase separation of thermo-responsive polymer solutions and precisely define the transition temperature ( $\left.\mathrm{T}_{\mathrm{PS}}\right)$. Our results are applied on hydroxypropylcellulose (HPC) solutions as an important biosourced green water-soluble polymer. As determined by DLS, the amplitudes of the fast and slow modes of relaxation dynamics evolve as temperature gets closer to the phase transition point eventually leading to phase separation. The evolution of the modes with temperature is markedly different for concentrations below the overlap concentration $\left(c^{*}\right)$ (dilute regime), above $c^{*}$ (semi-dilute regime) and above the entanglement concentration $\left(c_{e}\right)$. In the three cases though, the fast and slow mode amplitudes undergo a sharp transition in a narrow temperature range, defining accurately the phase separation locus. The results agree with turbidimetric analysis for the phase transition determination but with a better precision. Our results also show that the one-phase dynamics and phase separation dynamics in the twophase region are only in continuity for $c>c_{e}$, revealing mechanistic details about the HPC phase separation process. Above $\mathrm{T}_{\mathrm{PS}}$ we identify a temperature range where the intensity autocorrelation function has a single-exponential shape. In the latter regime, we monitor the 
growth kinetics of polymer domains and provide clues to rationalize the stabilizing effects of the interfaces leading to the arrested-like phase separation behavior observed for HPC.

\section{Introduction}

Phase separation of thermo-responsive water-soluble polymers is an intense research field in polymer science driven by promising technologies in a diverse range of fields, among biomedicine $^{1}$ and environmentally friendly materials..$^{2-4}$ Critical aspects to designing and screening such systems rely on a better understanding of the phase transition and the determination of well-characterized phase diagrams and phase transition solution temperatures since the latter temperatures are the main experimental data needed to further investigate the fundamentals of phase separation mechanisms and kinetics. Different approaches based on optical measurements (transmittance, scattering intensity at different angles and refractometry) and differential scanning calorimetry are currently utilized to approach and locate the phase boundary $\left(\mathrm{T}_{\mathrm{PS}}\right)$ of thermo-responsive water-soluble polymers in a broad range of concentrations. However, the typical criteria to define such transition are rather arbitrary without a clear physical significance that justifies a particular choice, which represents one of the major sources of diversity (as much as $20 \%$ of $\mathrm{T}_{\mathrm{PS}}$ ) of phase diagrams for several thermoresponsive polymers widely used in applications, such as poly( $N$-isopropylacrylamide $)$ $(\text { PNIPAm })^{5}$, hydroxypropylcellulose $(\mathrm{HPC})^{6,7}$, methylcellulose ${ }^{8-10}$, hydroxypropylmethyl cellulose $^{8,9}$, among others. Taking into account that phase separation mechanisms of thermoresponsive polymers strongly depend on the temperature quench depth ${ }^{10}$ the arbitrariness in defining $\mathrm{T}_{\mathrm{PS}}$ represents a clear limitation to investigating phase separation mechanisms and kinetics in a temperature range close to $\mathrm{T}_{\mathrm{PS}}$.

In this article we are interested in exploring another approach to define the phase separation transition of a thermo-responsive water-soluble polymer in a broad range of concentrations 
based on Dynamic Light Scattering (DLS). DLS is a well suited technique to investigate polymer dynamics over a large concentration range and a large temporal window of relaxation times. ${ }^{11}$ In the dilute regime, at which polymer concentration is below the overlap concentration $\left(c^{*}\right)$, the intensity autocorrelation function $\left(g_{2}(t)\right)$ reports a single relaxation mode which describes Brownian motion of single coils for monodisperse systems ${ }^{12}$ whatever the solvent quality is. ${ }^{13}$ At concentrations above $c^{*}$ the system resides in the semi-dilute regime, at which polymer chains overlap and possibly entangle. Li et al. demonstrated that for poly(styrene) in a thermodynamically good solvent (benzene) at $c / c^{*}=30, g_{2}(t)$ is still described by a single diffusive relaxation mode, ${ }^{14}$ while a second non-diffusive relaxation mode is evidenced (slow mode) at higher $t$ when the solvent quality gets poorer. ${ }^{13,14}$ The interpretation of the latter slow mode has been controversial and the subject of extensive research in the last decades for deciphering its origin with respect to blobs relaxations below or above the entanglement concentration $\left(c_{\mathrm{e}}\right)$. Pioneering works by Brown et al. ${ }^{15,16}$ and more recently Yuan et al. ${ }^{13}$ attributed the slow mode relaxation to permanent aggregates in the single-phase region, while more recent experiments ${ }^{11,17}$ described the slow relaxation dynamics of aqueous PNIPAm solutions as originating from transient clusters having a non-diffusive nature. In addition, a second slow dynamical mode was identified for shape-persisitent stiff polymers. ${ }^{18,19}$ Several studies in this area focused on the effect of polymer concentration, polymer architecture, and temperature on the slow mode ${ }^{17,20-22}$ in order to explain the origin of this slow relaxation dynamic. Here our goal is different: we address the question of whether it is possible to extract relevant quantitative information about the phase transition of a polymer solution in the semidilute regime by following the fast and slow mode evolution as temperature gets closer to phase separation. For this study we selected HPC aqueous solutions as a thermo-responsive biosourced polymer which was recently designed as an excellent candidate for making porous membranes excluding the use of organic solvents. ${ }^{3}$ It displays lower critical solution 
temperature behavior in water at $\sim 40^{\circ} \mathrm{C}$ (up to $40 \%$ ), ${ }^{23,24}$ a very convenient feature for many applications, and was first characterized in 1988 to phase separate by spinodal decomposition at $c=10 \% .^{25}$

In this article we show that the approach of HPC phase transition significantly impacts the fast and slow mode behaviors. By following the latter modes up to the phase boundary, we demonstrate that it is possible to define the phase transition temperature with a remarkable accuracy. This methodology provides a more precise and physically meaningful approach to determine the phase transition temperature than the commonly used turbidimetric method.

We also show that the evolution of relaxation modes for HPC is strongly concentration dependent and provides clues to describe the phase separation mechanisms by varying the concentration. For instance, a remarkable continuity of modes from the single-phase region to the two-phase region observed for entangled solutions reveals that the non-diffusive transient clusters formed in the single-phase region are precursors of the phase separating objects.

Finally, we show that DLS can be used to resolve the growth kinetics of HPC domains for concentrations as high as $5 \%$ and thermal quenches of an amplitude such as the single exponential nature of $g_{2}(t)$ is preserved.

\section{Experimental}

\section{Sample preparation}

A commercial HPC (Sigma-Aldrich) was employed for this study since the very same polymer proved to be a valuable choice for further applications for membranes. ${ }^{3}$ The weight average molecular weight $\left(M_{\mathrm{W}}\right)$, evaluated by size exclusion chromatography (Shimadzu LC-20AD) using a Shodex OHpak SB-803/SB-804 column and poly(ethylene glycol) molecular weight standards, is $72 \mathrm{~kg} / \mathrm{mol}$ and PDI 3. HPC aqueous solutions in the concentration range 0.5 to 30 $\%$ (wt.\%) were prepared by fully dispersing HPC powder in preheated Milli-Q water at $60^{\circ} \mathrm{C}$ 
with stirring for $2 \mathrm{~h}$. The samples were then cooled and stored at $4^{\circ} \mathrm{C}$ overnight to complete hydration of the polymer. The aqueous solutions were transparent at room temperature. Samples with $c \leq 10 \%$ were filtered using $0.45 \mu \mathrm{m}$ Millipore filters directly into dust-free PMMA light scattering cuvettes. Solutions with concentration higher than $20 \%$ were prepared from filtered $10 \%$ solution and concentrated under reduced pressure $(220 \mathrm{mbar})$ at $60^{\circ} \mathrm{C}$ due to the impossibility to filter such concentrated samples. The overlap concentration $c^{*}$ was determined from the definitions $c^{*}=3 M /\left(4 \pi N_{\mathrm{A}} R_{\mathrm{g}}^{3}\right), M /\left(2^{3 / 2} N_{\mathrm{A}} R_{\mathrm{g}}^{3}\right)$, and $[\eta]^{-1}$, where $M$, $R_{\mathrm{g}}, N_{\mathrm{A}}$ and $[\eta]$ are the molar mass, the radius of gyration of polymer chains, the Avogadro constant and the intrinsic viscosity, respectively. In this work we use the $c^{*}$ value obtained from $[\eta]^{-1}$, since the other two definitions are less precise due to the uncertainty in $R_{\mathrm{g}}$ as PDI is high. The entanglement concentration $c_{\mathrm{e}}$, representing the concentration above which the polymer chains form an entangled network, was estimated as $c_{\mathrm{e}} \approx 10 c^{*}$, as reported by Colby ${ }^{26}$ for neutral polymers in good solvent conditions. The radius of gyration of polymer chains was determined as $R_{\mathrm{g}}=1.56 R_{\mathrm{h}}$, assuming good solvent conditions far enough from $\mathrm{T}_{\mathrm{PS}}$, where $R_{\mathrm{h}}$ is the hydrodynamic radius calculated from diffusion coefficient $D$ obtained by DLS experiments of diluted samples employing the Stokes-Einstein equation, $R_{\mathrm{h}}=\left(k_{\mathrm{B}} T / 6 \pi \eta_{0}\right) / D$, where $k_{\mathrm{B}}, T$ and $\eta_{0}$ are the Boltzmann constant, the absolute temperature, and the solvent viscosity, respectively. Since the polymer is polydisperse, $D$ means actually an average $D$ over the size distribution of chains. Intrinsic viscosity was measured by the rolling ball principle using a microviscometer Lovis 2000M (Anton Paar) at a fixed angle of $85^{\circ}$. HPC solutions were prepared by dilution in the concentration range of $0.01-2 \%$ and measured at $30^{\circ} \mathrm{C}$.

\section{Dynamic light scattering}

Back-scattering intensity autocorrelation function $\left(g_{2}(t)\right)$ was obtained using a Zetasizer Nano ZS (Malvern Instruments Ltd., Worcestershire, UK) (temperature control range $0-90^{\circ} \mathrm{C}, \pm$ 
$0.1^{\circ} \mathrm{C}$ ) equipped with a $4 \mathrm{~mW}$ He-Ne laser of $\lambda_{\mathrm{o}}=633 \mathrm{~nm}$. The angle between the laser beam and the detector (avalanche photodiode) was $\theta=173^{\circ}$ and the scattering vector $(q)(q=$ $\left.\left(4 \pi n / \lambda_{0}\right) \sin (\theta / 2)\right)$ was $0.02633 \mathrm{~nm}^{-1}$. The laser power was automatically attenuated to collect an optimal scattered intensity. The measurement penetration depth into the sample was set to $2 \mathrm{~mm}$. A $30 \mathrm{~s}$ acquisition time was generally enough to obtain a stable intensity autocorrelation function. Increasing the acquisition time up to 100 times the slower dynamical mode relaxation time did not provide any additional change in the signal of the intensity autocorrelation function. HPC solutions were heated at different $\mathrm{T}$ in the range between 29.4 and $48^{\circ} \mathrm{C}$ in which $T_{P S}$ is found. The heating rate $\left(3^{\circ} \mathrm{C} / \mathrm{min}\right)$ allowed a fast thermal equilibration at each final temperature (equilibration time was measured using a thermocouple within solution). Measurements in the two-phase region ( $\mathrm{T}>\mathrm{T} \mathrm{PS})$ were performed either close to $\mathrm{T}_{\mathrm{PS}}\left(\mathrm{T}-\mathrm{T}_{\mathrm{PS}} \leq 4{ }^{\circ} \mathrm{C}\right)$ or well above $\mathrm{T}_{\mathrm{PS}}\left(\mathrm{T}-\mathrm{T}_{\mathrm{PS}} \geq 10^{\circ} \mathrm{C}\right)$. The final temperature was reached after 1 min heating in the two-phase region at T close to TPS and up to 4 min heating in the two-phase region at $\mathrm{T}$ well above to $\mathrm{T}_{\mathrm{PS}}$. Intensity autocorrelation functions were measured at different temperatures as a function of time at 5 min time intervals until no significant differences were observed in consecutive measurements (between 10-30 min). Repeated measurements were performed at each temperature at $1 \mathrm{~h}$ interval to ensure data are in steady state. Before each measurement at a certain temperature, an equilibration step at $29.4^{\circ} \mathrm{C}$ for 30 min was performed.

$g_{2}(t)$ can be related to the normalized electric field correlation function $g_{1}(t)$ by the Siegert relation as ${ }^{12}$

$$
g_{2}(t)=\beta\left|g_{1}(t)\right|^{2}
$$

where $0<\beta<1$ is a constant related to the coherence of the detection optics. For a polydisperse system, ${ }^{27} g_{1}(t)$ is related to the distribution of the characteristic relaxation time distribution $(G(\tau))$ as 


$$
\left|g_{1}(t)\right|=\int_{0}^{\infty} G(\tau) e^{-t / \tau} \mathrm{d} \tau
$$

In this study $(G(\tau))$ was calculated using the Laplace inversion of $g_{1}(t)$ (normalized to 1 at $t=0$ ) on the basis of eqs 1 and 2 by the Maximum Entropy Method. ${ }^{12,28} G(\tau)$ usually displays two major relaxation modes (fast and slow). Fast mode correlation time $\left(\tau_{\mathrm{fm}}\right)$ and slow mode correlation time $\left(\tau_{\mathrm{sm}}\right)$ were extracted from the mean peak position of fast and slow relaxation modes, respectively. The contribution of each mode (amplitude) was obtained from the relative peak area of each distribution mode. Additionally, correlation times and amplitudes were also obtained by a similar analysis than that reported by Yamamoto et al. ${ }^{20}$ by fitting to a sum of single-exponential functions. The obtained results (data not shown) were in good agreement with those obtained from Maximum Entropy Method.

\section{Turbidimetric measurements}

Phase separation temperatures were determined by optical transmittance method using a quartz cell filled with HPC solutions inserted in a thermostat $\left(0.01^{\circ} \mathrm{C}\right.$ precision $)$. For determining $\mathrm{T}_{\mathrm{PS}}$, a $5 \mathrm{~mm}$ thick cell was used, shined by a laser beam $(\lambda=632.8 \mathrm{~nm})$. Two photodiodes were placed before and after the sample to measure the transmission of the light through the sample. Thermal steps of $0.2^{\circ} \mathrm{C}$ followed by 30 min equilibration time were performed to monitor the transmittance versus time. As the temperature approached $T_{P S}$ the transmittance decreased with time asymptotically, and therefore the transmittance at infinite time $\tau_{\infty}\left(\mathrm{T}_{\infty}\right)$ at each thermal step was extrapolated by fitting to $y=A_{1} \exp ^{\left(-t / t_{1}\right)}+\mathrm{T}_{\infty}$. From transmittance vs temperature curves, TPS was determined by two different criteria: i) As the abscissa of the intercept between the horizontal asymptote at low temperatures and the tangent to the transmission decrease $\left(\mathrm{T}_{-}\right)$; ii) As the middle point on the slope of variation between $\tau_{\infty}$ and $\tau_{0}\left(\mathrm{~T}_{1 / 2}\right)$. 


\section{Confocal laser scanning microscopy}

HPC phase separation was monitored using an Olympus Fluoview FV1000 inverted confocal microscope. HPC solution $(\sim 10 \mu \mathrm{L})$ was sandwiched between two glass slides separated and sealed by a polydimethylsiloxane ring ( $200 \mu \mathrm{m}$ thick). Rhodamine $6 \mathrm{G}$ Chloride was used as the hydrophilic fluorophore. Micrographs were collected with a 40X objective. A thermal stage (Linkam PE 94) was used to control the temperature. The solutions were equilibrated at $30^{\circ} \mathrm{C}$ for $30 \mathrm{~min}$ followed by heating to the final quench temperature at a rate of $5^{\circ} \mathrm{C} / \mathrm{min}$. The working distance of the objective was focused in a plane inside the solution, away $(\sim 40 \mu \mathrm{m})$ from the cover glass, in order to avoid interface effects.

\section{Results and discussion}

The overlap concentration $\left(c^{*}\right)$ was estimated to be $1.2 \%$ as defined in the experimental section. In the dilute regime the product $q . R_{\mathrm{g}}$, where $q$ is the scattering vector and $R_{\mathrm{g}}$ the radius of gyration of the polymer, was estimated to be $0.592\left(q=0.02633 \mathrm{~nm}^{-1}, R_{\mathrm{g}}=22 \mathrm{~nm}\right)$ satisfying $q . R_{\mathrm{g}}<1$. Under these experimental conditions the correlation function yields information about the whole macromolecular motion and not about internal motions of single coils. ${ }^{29}$ At $c=0.5 \%$ the system is in the dilute regime and $\mathrm{T}_{\mathrm{PS}}$ was determined to be $\sim 44^{\circ} \mathrm{C}$ from turbidimetric measurements. The entanglement concentration $c_{\mathrm{e}}$ for aqueous $\mathrm{HPC}$ at $30^{\circ} \mathrm{C}$ should be close to $10 \%$ based on $c^{*}$ value. Figure 1 shows the intensity-intensity time correlation function $g_{2}(t)$ variation in the T range between 29.4 and $47.3^{\circ} \mathrm{C}$ for $\mathrm{HPC}$ aqueous solutions in the dilute regime $(c=0.5 \%)$ (Figure 1a) and semi-dilute regime above $c_{\mathrm{e}}(c=$ $20 \%$ ) (Figure 1b) collected after 30 min equilibration. By increasing $\mathrm{T}$ in a narrow temperature range $\left(42.8-43.8{ }^{\circ} \mathrm{C}\right.$ at $0.5 \%$ and $36.5-37.6{ }^{\circ} \mathrm{C}$ at $\left.20 \%\right)$ the $g_{2}(t)$ signal presents an abrupt change, corresponding to the phase separation transition. Decay time distribution functions 
$(G(\tau))$ were obtained from the Laplace inversion by the Maximum Entropy Method. The temperature evolution of $G(\tau)$ at different concentrations is presented in Figure 2 where the relaxation time distribution is plotted as a function of $\tau T / \eta_{0}$ at different polymer concentrations and temperatures, where $\eta_{0}$ is the solvent viscosity at each $\mathrm{T}$. This renormalization of time was adopted to suppress trivial thermal dependence not related to phase separation, enabling direct comparison of peak position at different $\mathrm{T} .^{14,15,30}$
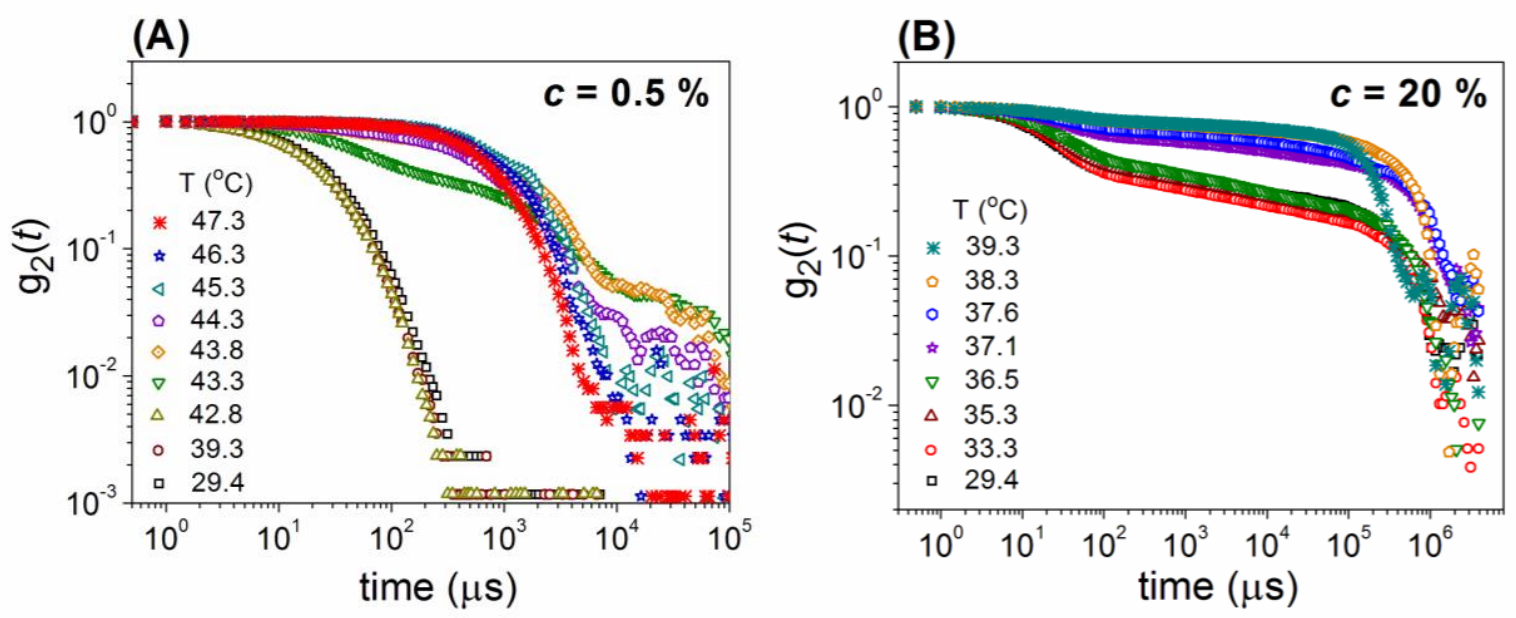

Figure 1. Temperature dependence of intensity autocorrelation function $g_{2}(\tau)$ for concentrations (a) $0.5 \%$ and (b) $20 \%$ at a scattering angle of $173^{\circ}$.

At $c=0.5 \% G(\tau)$ reports a single relaxation mode (at $\tau T / \eta_{0} \sim 27 \mathrm{~Pa}^{-1} \mathrm{~K}$ ) below $42.8^{\circ} \mathrm{C}$, which turns into a single relaxation mode with higher $\tau T / \eta_{0}\left(\sim 10^{3} \mathrm{~Pa}^{-1} \mathrm{~K}\right)$ from $43.8^{\circ} \mathrm{C}$, as the polymer phase separates (Figure 2a). It is worth noting that at $c<c^{*}$ in the single-phase region $(\mathrm{T} \leq$ $42.8^{\circ} \mathrm{C}$ ) the distribution observed for the single relaxation mode is rather broad as a result of the polydisperse nature of the biosourced commercial HPC. By increasing $c$ to $1 \%$ (roughly at $\left.c^{*}\right)$, turbidimetric analysis estimates a $\mathrm{T}_{\mathrm{PS}}$ of $42.7^{\circ} \mathrm{C}$. Figure $2 \mathrm{~b}$ shows that relaxation time distribution at $c=1 \%$ displays a principal mode $\left(\tau T / \eta_{0} \sim 20 \mathrm{~Pa}^{-1} \mathrm{~K}\right)$ together with a slow 
mode of small amplitude at higher $\tau T / \eta_{0}\left(\sim 100 \mathrm{~Pa}^{-1} \mathrm{~K}\right)$. Fast and slow modes are almost constant in reduced $\tau$ by increasing $\mathrm{T}$ in the range $(29.4-41.3)^{\circ} \mathrm{C}$. However, in the narrow $\mathrm{T}$ range $41.3-43.3^{\circ} \mathrm{C}$ around $\mathrm{T}_{\mathrm{PS}}$ the proportion of the fast and slow mode inverts showing a marked shift in amplitudes downwards and upwards, respectively. Additionally, the slow mode increases in $\tau T / \eta_{0}$ by a noticeable amount at the transition.
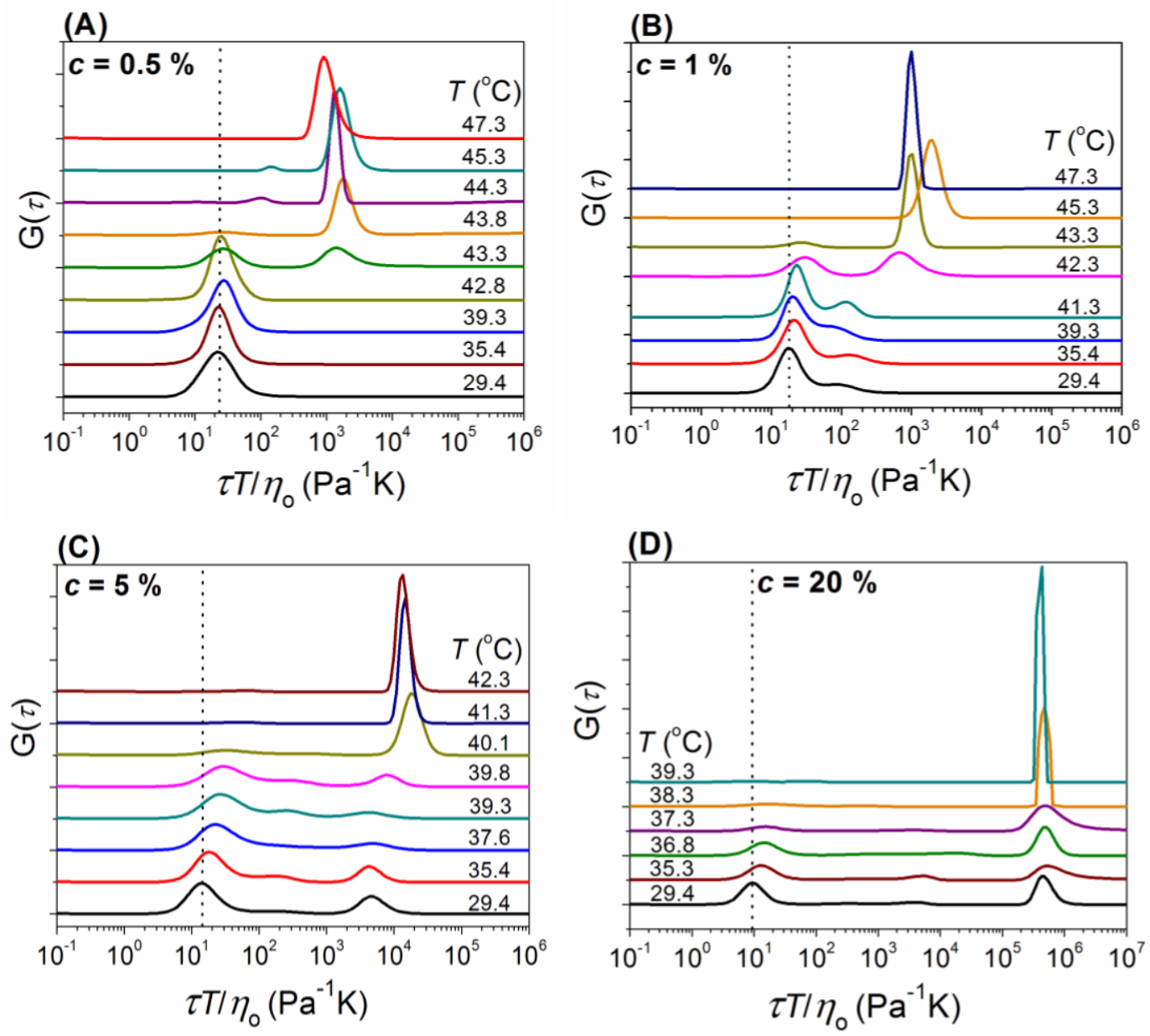

Figure 2. Relaxation time distribution $G(\tau)$ at different temperatures for concentrations (a) $0.5 \%$, , b) $1 \%$, (c) $5 \%$ and (d) $20 \%$. 
At $c \geq 5 \%$ the complexity of $G(\tau)$ increases. Two major modes (fast and slow) are observed in combination with a small contribution of a third mode at $\tau T / \eta_{0}$ between fast and slow modes. However, due to the negligible contribution of this third mode, we shall ignore this intermediate relaxation mode in the following analysis. A first qualitative analysis of relaxation mode evolution with $\mathrm{T}$ in the semi-dilute regime indicates that at $c=5 \%\left(\mathrm{~T}_{\mathrm{PS}} \sim 40^{\circ} \mathrm{C}\right.$ by turbidimetry $)$ the fast and slow mode contributions are equivalent and constant with increasing $\mathrm{T}$ until a narrow temperature range $(39.3-40.1)^{\circ} \mathrm{C}$ at which the fast mode decreases to almost zero and the slow mode amplitude increases sharply, as presented in Figure 2c. Moreover, reduced relaxation time of fast mode undergoes a slight shift to higher $\tau T / \eta_{0}$ in the range $29.4-39.8^{\circ} \mathrm{C}$, while that for the slow mode is roughly constant in the same T range. However, the slow mode undergoes a marked shift to higher $\tau T / \eta_{0}$ in the T range (39.3-40.1) ${ }^{\circ} \mathrm{C}$ close to $\mathrm{T}_{\mathrm{PS}}$.

At $c=20 \%$ (Figure $2 \mathrm{~d}$ ) the behavior of the fast mode is similar to that observed at $c=5 \%$, showing a marked shift to higher $\tau T / \eta_{0}$ with increasing $\mathrm{T}$. However, the slow mode now displays a remarkable continuity in $\tau T / \eta_{0}$ in the entire $T$ range analyzed in this study. Interestingly, at all concentrations it is observed that above the temperature range at which the fast mode vanishes i.e. in the two-phase region, $G(\tau)$ turns out to be a single narrow mode related to phase separation under the form of polymer aggregates. Small variations in normalized $\tau$ at this $\mathrm{T}$ range are likely due to the aggregate size dependence on the thermal quench, an effect consistent with previous phase separation studies on HPC ${ }^{25}$ and PNIPAm. ${ }^{31}$

\section{Fast mode}

When the polymer concentration is below $c^{*}$ the single relaxation mode is attributed to Brownian motion of single coils, ${ }^{12}$ whereas for concentrations above the overlap concentration $\left(c>c^{*}\right)$ this mode reflects cooperative diffusion of chain segments between each blob. ${ }^{32}$ Figure 3a shows the polymer concentration dependence of fast mode relaxation time at $\mathrm{T}=29.4$ 
${ }^{\circ} \mathrm{C}$. An approximate plateau region is found at concentrations below $0.5 \%$ whereas $\tau_{\mathrm{fm}}$ decreases more markedly with polymer concentration above $0.5 \%$. This observation suggests that the solution below $0.5 \%$ is in the dilute regime $\left(c<c^{*}\right)$ while at $c>1 \%$ the system enters the semi-dilute regime, in agreement with $c^{*}$ estimation $(\sim 1.2 \%)$. The decrease in $\tau_{\mathrm{fm}}$ as the polymer concentration increases above $1 \%$ indicates that the average segment correlation length decreases as concentration increases, following a similar trend as that reported for PNIPAm. ${ }^{33}$
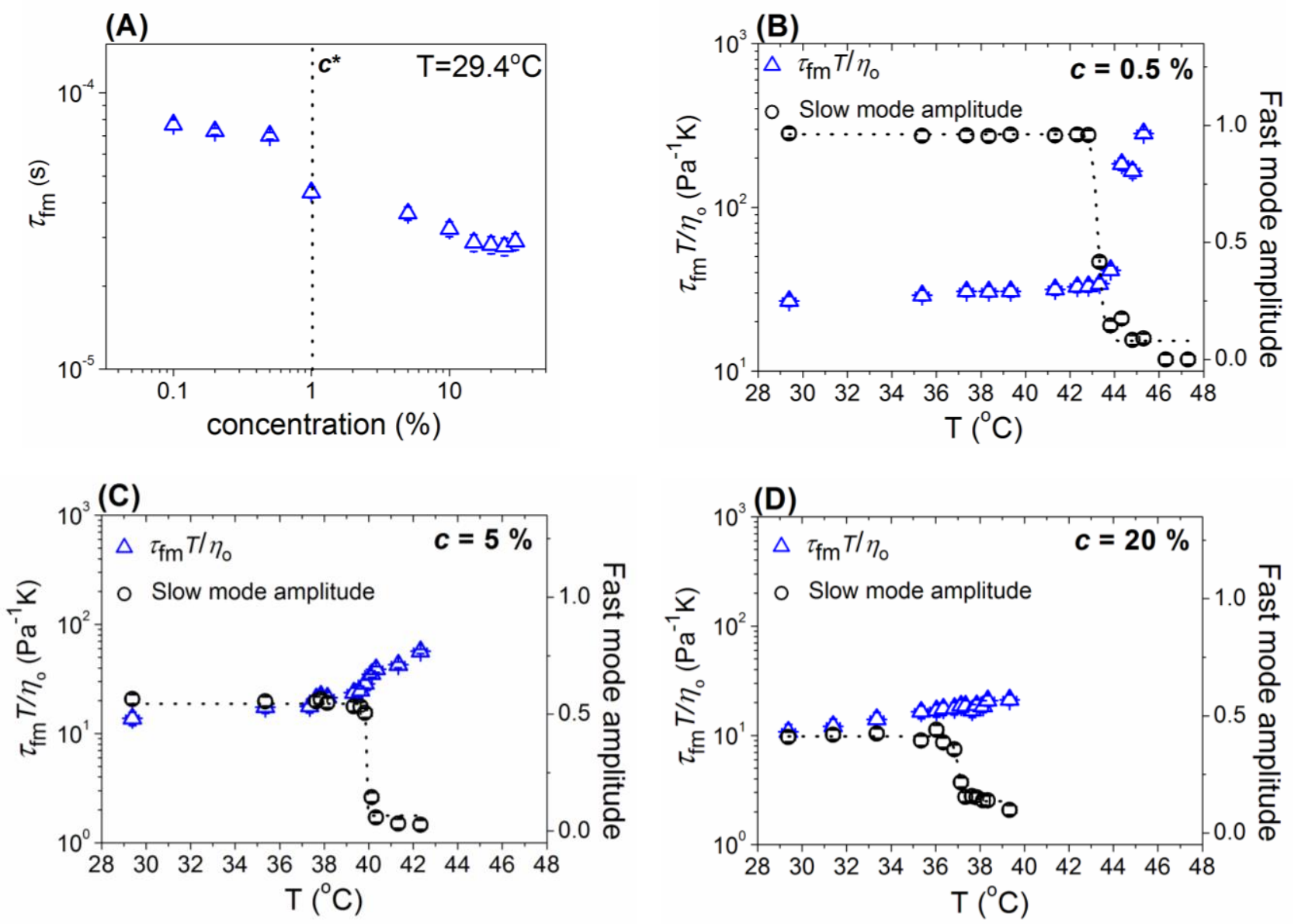

Figure 3. (a) Fast mode relaxation time vs. concentration in the single phase region at $29.4^{\circ} \mathrm{C}$ and normalized fast mode relaxation time $\left(\tau_{\mathrm{fm}} T / \eta_{0}\right)$ vs. $\mathrm{T}$ for concentrations (b) $0.5 \%$, (c) 5 $\%$ and (d) $20 \%$. 
Figures 3b-d display the temperature dependence of the reduced fast mode relaxation time $\left(\tau_{\mathrm{fm}} T / \eta_{0}\right)$ at three different concentrations $(0.5,5$ and $20 \%)$. As discussed before, when the polymer phase separates by increasing $\mathrm{T}$ the slow mode becomes the major distribution and the fast mode contribution decreases to almost zero. However, in a temperature range above the transition but close enough to it, the decay time distribution functions obtained by the Maximum Entropy Method still evidence a small contribution of fast mode that is reported in Figure 3. At $c=0.5 \%, \tau_{\mathrm{fm}} T / \eta_{0}$ undergoes a slight increase with $\mathrm{T}$ up to $\sim 43^{\circ} \mathrm{C}$ above which it displays an abrupt shift to higher values as the fast mode disappears. Reduced relaxation time at $c=5 \%$ shows a slight increase with $\mathrm{T}$ below $39^{\circ} \mathrm{C}$, which becomes more notorious above $39^{\circ} \mathrm{C}$, likely due to phase separation (PS) into a polymer rich and a polymer lean phase. However, the latter increment of $\tau_{\mathrm{fm}} T / \eta_{0}$ in the two-phase region at $c=5 \%$ is notably less marked than that at $c=0.5 \%$. On the contrary, at $c=20 \%$ the reduced relaxation time $\tau_{\mathrm{fm}} T / \eta_{0}$ steadily increases with $\mathrm{T}$, but no sharp transition is observed within the analyzed temperature range. The slight increment of $\tau_{\mathrm{fm}} T / \eta_{0}$ observed below PS in the entire concentration range is likely due to the gradual decrease in solvent quality as the temperature increases from $30^{\circ} \mathrm{C}$ (relatively good solvent condition) to the vicinity of theta condition $\left(\sim 40^{\circ} \mathrm{C}\right)^{34}$ where the interactions between segments and segment-solvent gradually change. Brown et al. showed a similar slight increase of fast relaxation time for polystyrene semi-dilute solutions from good solvent (toluene) to theta solvent (2-butanone) conditions, while the fast mode amplitude remained constant. ${ }^{29} \mathrm{~A}$ similar trend was observed by Li et al. ${ }^{14}$ by cooling polystyrene solution in cyclohexane. The authors explained these results by considering that when the solvent quality decreases, polymer chains contract, resulting in an increase of $c^{*}$ and therefore a slight shift in fast mode relaxation time to higher values, as shown in Figure 3a. Interestingly, over the entire concentration range studied here the fast mode amplitude remains constant up to a temperature at which it decreases sharply, which is likely due to phase separation transition. Also, our results 
show that the temperature variation of the reduced fast mode relaxation time has no clear dependence with the concentration ranges delimited by $c^{*}$ or $c_{\mathrm{e}}$.

\section{Slow mode}

Figure $4 \mathrm{a}$ shows the concentration dependence of the reduced slow mode relaxation time $\left(\tau_{\mathrm{sm}} T / \eta_{0}\right)$ at $29.4^{\circ} \mathrm{C}$ in the single phase region and the corresponding amplitude. As the concentration increases, $\tau_{\mathrm{sm}} T / \eta_{0}$ is larger and the slow mode amplitude increases. These observations indicate that the slow dynamic process is closely related to chain clustering/entanglement effects. ${ }^{17}$ DLS experiments in the forward-scattering configuration at $\mathrm{c}=20 \%\left(\theta=13^{\circ} ; q^{2}=8.925 \times 10^{-6} \mathrm{~nm}^{-2}\right.$, acquisition time $\left.1000 \mathrm{~s}\right)$ provided values of $\tau_{\mathrm{sm}}=29.3 \mathrm{~s}$ and fast mode amplitude/slow mode amplitude $=0.59$, while the corresponding values for back-scattering $\left(\theta=173^{\circ} ; q^{2}=6.9327 \times 10^{-4} \mathrm{~nm}^{-2}\right)$ were $1.2 \mathrm{~s}$ and 1.05 , respectively. The plot $1 / \tau_{\text {sm }}$ vs. $q^{2}$ deviates from a straight line passing through the origin, evidencing the non-diffusive nature of the slow mode as previously observed for different polymer solutions. ${ }^{13}$ The plots of the slow mode amplitude vs T shown in Figure $4 \mathrm{~b}-\mathrm{f}$ reveal that at some temperature the amplitude undergoes a sharp transition to higher values in the entire concentration range between 1 and $30 \%$. Moreover, the temperature of this transition decreases with polymer concentration and coincides with the fast mode amplitude transition. Therefore, we can now rationalize the fast and slow amplitude shifts with increasing temperature as coinciding with the HPC phase separation transition, where polymer chains collapse into polymer aggregates. 

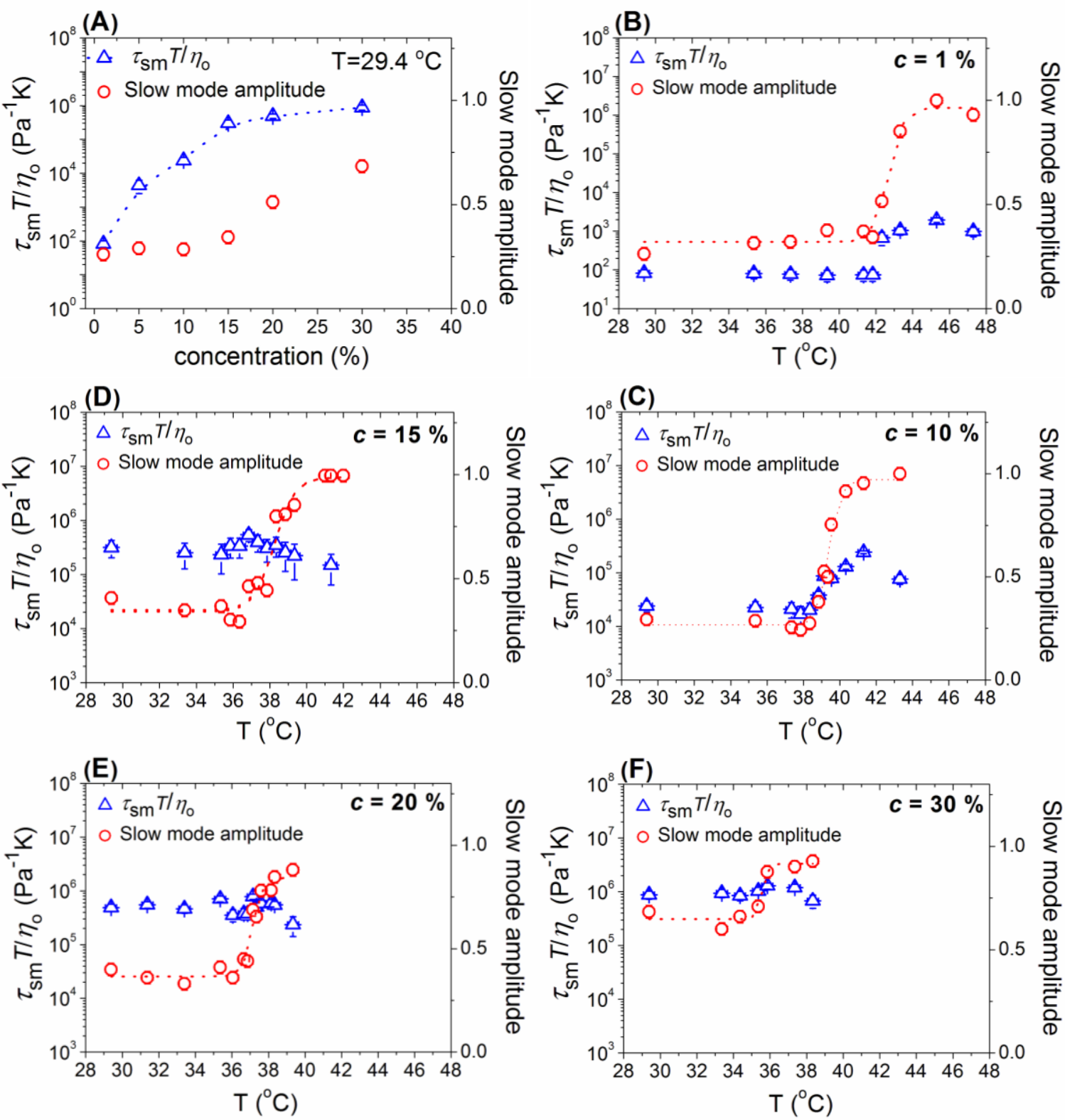

Figure 4. (a) Reduced slow mode relaxation time $\left(\tau_{\mathrm{sm}} T / \eta_{0}\right)$ and slow mode amplitude vs. concentration in the single-phase region at $29.4^{\circ} \mathrm{C}$. Reduced slow mode relaxation time and amplitude dependence on T for concentrations (b) $1 \%$, (c) $10 \%$, (d) $15 \%$, (e) $20 \%$ and (f) 30 $\%$. Dotted lines correspond to sigmoidal fittings for amplitude vs T plots.

Note that at $c \leq 10 \%$ there is a narrow T range at which $\tau_{\mathrm{sm}} T / \eta_{0}$ shifts to larger correlation times, as presented in Figure 4b-d. This temperature range coincides with the temperature at which the slow mode amplitude increases sharply and is the evidence of HPC demixing process. 
This behavior is consistent with results by Yamamoto et al. ${ }^{20}$ for semi-dilute aqueous PNIPAm solutions showing a sharp transition in slow mode relaxation time at $\mathrm{T} \sim \mathrm{T}_{\mathrm{PS}}$. However, it is worth highlighting that the slow mode behavior with increasing temperature observed for HPC is in marked contrast to that reported by Yuan et al. for aqueous PNIPAm in semi-dilute regime, where the slow mode reduced $\tau$ was observed to become faster by increasing $\mathrm{T}$ below $\mathrm{T}_{\mathrm{PS}}{ }^{13}$ This suggests that the slow mode reduced $\tau$ variations with temperature could be dependent on the nature of the thermo-responsive polymer. Remarkably, this behavior is no longer observed for HPC solutions above $c=15 \%$ as the reduced $\tau_{\text {sm }}$ shows a clear continuity before and after phase separation, as presented in Figures 4e,f. In this regard, we suggest that the different T dependence of $\tau_{\mathrm{sm}} T / \eta_{0}$ at $c \leq 10 \%$ and at $c \geq 15 \%$ is related with the transition between the overlap and entanglement regimes ( $c_{\mathrm{e}}$ is roughly $\left.10 \%\right)$. In the non-entangled range $\left(c^{*}<\right.$ $c \leq c_{\mathrm{e}}$ ), at which polymer chains overlap to some extent without entanglement formation, the shift of $\tau_{\mathrm{sm}} T / \eta_{0}$ to higher values by increasing $\mathrm{T}$ at $\sim \mathrm{T}_{\mathrm{PS}}$ reflects chain and clustering association at the phase separation condition. By contrast, in the entangled range $\left(c>c_{\mathrm{e}}\right)$ the fact that $\tau_{\mathrm{sm}} T / \eta_{0}$ is constant below and above $\mathrm{T}_{\mathrm{PS}}$ would imply that no additional or further chain/cluster association occurs during phase separation in this concentration range. This presumably reflects that the transient clusters present in the single-phase region are precursors of polymer aggregates formed in the two-phase region. This picture of phase separation of HPC aqueous solutions at $c>c_{\mathrm{e}}$ raises the question of the nature of the molecular organization occurring at the phase separation transition. While the observation that the fast mode amplitude decreases abruptly at $\mathrm{T}_{\mathrm{PS}}$ reflects the formation of HPC-HPC contacts/interaction, the almost identical relaxation dynamics found between the slow mode below $\mathrm{T}_{\mathrm{PS}}$ and the single mode above $T_{P S}$ suggest that preformed aggregates in the two-phase region may retain considerable amount of hydrogen bonded water molecules, as recently described by Patra et al. ${ }^{35}$ 


\section{Phase diagram}

From the analysis of fast and slow correlation times and amplitudes it was evidenced that PS temperatures at different concentrations cannot be obtained by following the evolution of the reduced relaxation times only, in particular at $c>c_{\mathrm{e}}$. Although this approach could be useful in the dilute regime, ${ }^{9}$ the lack of sharpness in temperature dependence when the concentration is above $5 \%$ precludes a precise definition of the phase separation transitions.

By contrast, the observed transitions in the fast and slow mode amplitudes could be employed to precisely map phase separation diagram of HPC in a broad concentration range and this approach compares favorably to $\mathrm{T}_{\mathrm{PS}}$ determination obtained by other methods. For comparison, Figure 5a shows that the phase separation temperatures determined by the fast and slow mode amplitude evolution with $\mathrm{T}$ are identical. The advantage of this approach is that this DLS analysis conducts to sharp transitions that can be used to accurately define $\mathrm{T}_{\mathrm{PS}}$ as the temperature at which the fast and slow mode amplitude diverges (experimental error $\pm 0.3^{\circ} \mathrm{C}$ ). On the other hand, the typical method employed to map phase separation diagrams of HPC, based on following the drop in transmittance as the polymer phase separates with increasing temperature, provides a transmittance signal that decreases slowly with $\mathrm{T}$ (Figure 5a). The latter approach prevents a well-defined $\mathrm{T}_{\mathrm{PS}}$ determination because the transition lacks sufficient sharpness, reducing the accuracy of the method. ${ }^{6}$ In fact, this drawback is one of the main factors contributing to diversity of phase separation diagrams since different criterions can be selected to determine $\mathrm{T}_{\mathrm{PS}}$, as outlined in a recent work of Halperin et al. $^{5}$ In this regard, a significant discrepancy (see Figure 3 in Marsano et al..$^{7}$ ) was found by comparing previously reported phase diagrams of aqueous HPC based on turbidity measurements from HPC with similar molecular weight and structure for which no discrepancy is expected. ${ }^{6,36}$ Figure $5 \mathrm{~b}$ displays the phase separation diagrams for aqueous HPC solutions in the concentration range $c=(0.5-30) \%$ obtained by DLS from sigmoidal fittings of fast and slow mode amplitude 
transitions (red) and turbidimetric analysis taken as the midpoint of the transition $\left(\mathrm{T}_{1 / 2}\right)$ (green) and by the tangent method ( $\mathrm{T}_{-}$) (blue). We found that $\mathrm{T}_{\mathrm{PS}}$ obtained by DLS are in very good agreement with optical transmittance results $T_{-}$but with much smaller error bars for the DLS determination. By contrast, the $\mathrm{T}_{1 / 2}$ method appears to exceed $\mathrm{T}_{\mathrm{PS}}$ obtained by DLS at equivalent polymer concentration by an average of $2.0^{\circ} \mathrm{C}$ (experimental error $\pm 0.3^{\circ} \mathrm{C}$ ), which becomes even larger at the lowest concentration $\left(\mathrm{T}_{1 / 2}-\mathrm{T}_{\mathrm{PS}}=4.5^{\circ} \mathrm{C}\right)$. However, the general trend of the diagrams is very similar with a pronounced decrease of $\mathrm{T}_{\mathrm{PS}}$ at $0.5 \%<c<1 \%$ and a gradual decrease at $1 \%<c<30 \%$, in agreement with some previous theoretical and experimental HPC phase diagrams reported by Lárez-V et al. ${ }^{37}$ This approach thanks to DLS fully clarifies why the so far rather empirical choice of $\mathrm{T}_{-}$as the PS temperature is probably justified but much less precise.

Additional experiments performed on aqueous poly(vinyl)alcohol solutions (10 wt.\%) with a degree of hydrolysis of $72 \%$ also showed good agreement between the TPS obtained by DLS $\left(36^{\circ} \mathrm{C}\right.$, data not shown) and the onset of phase separation previously reported by turbidimetric analysis, ${ }^{2}$ suggesting that the method to track the $\mathrm{T}_{\mathrm{PS}}$ presented here is probably relevant for many thermo-responsive water-soluble polymer solutions in the semi-dilute regime. 

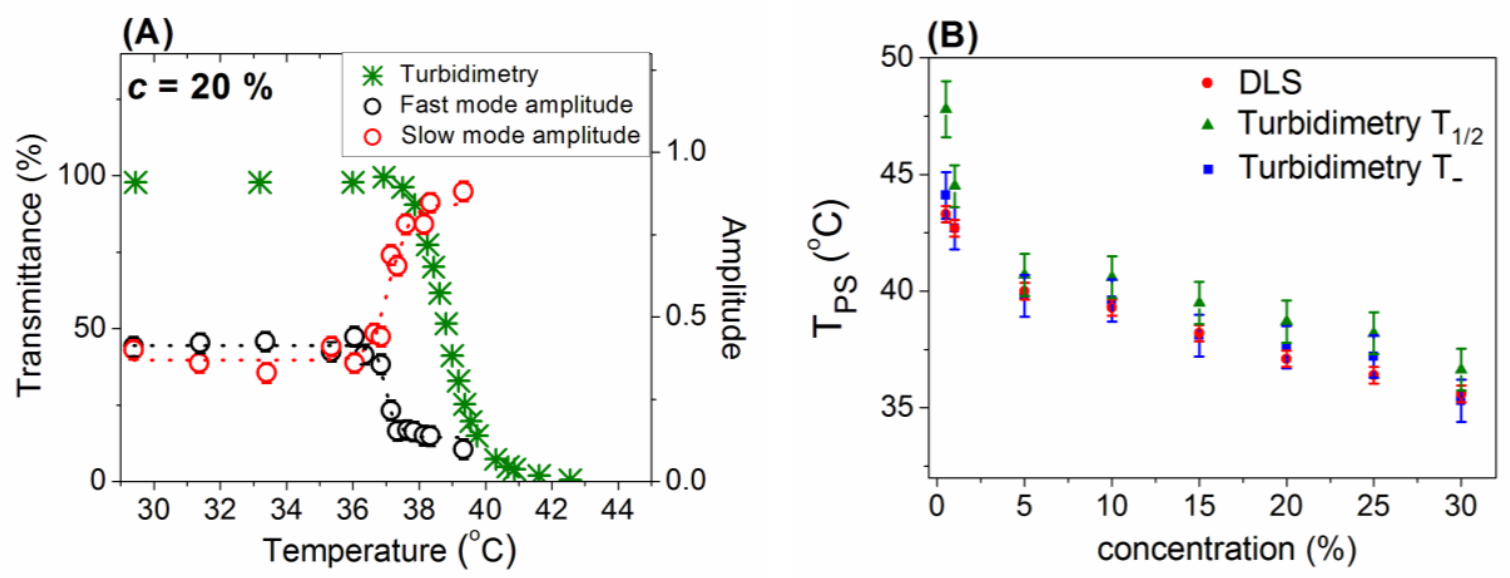

Figure 5. (a) Transmittance, fast and slow mode amplitude temperature dependence for $c=$ $20 \%$. Dotted curves correspond to sigmoidal fittings. (b) Phase diagram of aqueous HPC obtained by DLS and turbidimetry $\left(\mathrm{T}_{-}, \mathrm{T}_{1 / 2}\right)$ in the range $c=(0.5-30) \%$.

\section{Insights into the two-phase region}

A correct interpretation of $g_{2}(t)$ in the two-phase region may give access to monitoring the phase separation kinetics and measuring characteristic size of polymer aggregates which may form and grow with time. However, DLS theory can only be applied to interpret $g_{2}(t)$ signal provided multiple-scattering effects are absent. As HPC aqueous solution enters into the twophase region, the system gets turbid (near $\mathrm{T}_{\mathrm{PS}}$ ) and by further heating the sample well above $\mathrm{T}_{\mathrm{PS}}\left(\mathrm{T}-\mathrm{T}_{\mathrm{PS}} \geq 10^{\circ} \mathrm{C}\right)$, it turns into a cloudy phase separated system. Despite this apparent multiple-scattering character above $\mathrm{T}_{\mathrm{PS}}$, the correct interpretation of $g_{2}(t)$ actually depends on the T quench depth. To illustrate this effect, $g_{2}(t)$ was collected at different temperatures using a heating rate of $3{ }^{\circ} \mathrm{C} / \mathrm{min}$ to allow for a fast quenching experiment (less than 1 or $4 \mathrm{~min}$ heating in the two-phase region for the lower and higher $\mathrm{T}$ quench, respectively). Figure 6a shows that $g_{2}(t)$ signal is mostly a single exponential in the two-phase region near $\mathrm{T}_{\mathrm{PS}}(\mathrm{T}-$ $\left.\mathrm{T}_{\mathrm{PS}} \leq 4{ }^{\circ} \mathrm{C}\right)$. By contrast, for a higher $\mathrm{T}$ quench $\left(\mathrm{T}-\mathrm{T}_{\mathrm{PS}} \geq 10^{\circ} \mathrm{C}\right), g_{2}(t)$ deviates from the single exponential behavior, as represented in Figure 6a at the same concentration. This is likely 
due to the formation of denser polymer aggregates that act as efficient scatterers bringing multiple-scattering effects. Indeed, in the multiple-scattering regime, in the back-scattering geometry, $g_{2}(t)$ can be fitted to Eq. 3 according to diffusing-wave spectroscopy theory (DWS) assuming spherical scatterers. ${ }^{38}$

$$
g_{2}(t)=e^{-2 \gamma\left(\frac{6 t}{t_{0}}\right)^{a}}(a=0.5)
$$

with $\gamma=\frac{\left\langle z_{o}\right\rangle}{l^{*}}+\frac{2}{3}$, where $2 / 3$ is an empirical fitting parameter, $l^{*}$ is the transport mean free path and $\left\langle z_{o}\right\rangle$ is the average penetration depth into the sample (2 $\mathrm{mm}$ in the experiments), $t_{0}$ is the characteristic relaxation time and the exponent $a$ is 0.5 in DWS theory. The $g_{2}(t)$ signal was measured as a function of time at $50^{\circ} \mathrm{C}$ for concentrations 5,10 and $20 \%$ and fitted to $\mathrm{Eq} 3$. At $30 \mathrm{~min}$, the best-fitted values of $a$ are $0.65,0.51$ and $0.49( \pm 0.03)$ at 5,10 and $20 \%$, respectively. Figure $6 \mathrm{~b}$ displays the logarithm of the normalized autocorrelation function at $c=$ $5 \%, 10 \%$ and $20 \%$ plotted as a function of $\left(t / t_{0}\right)^{a}$ using the best values of $a$.

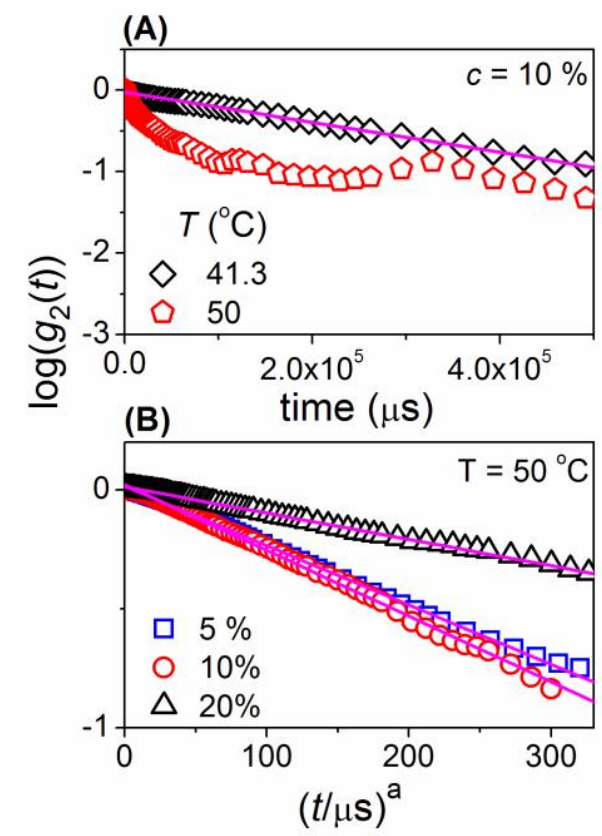

Figure 6. $\log \left(g_{2}(t)\right)$ collected in the back-scattering geometry $\left(173^{\circ}\right)$ after 30 min equilibration. Data is plotted as function of time (a) at $41.3^{\circ} \mathrm{C}$ (diamonds) and $50^{\circ} \mathrm{C}$ (pentagons) 
$(c=10 \%)$ and as a function of $\left(t / t_{0}\right)^{a}$ (b) at $50^{\circ} \mathrm{C}$ at concentrations $5 \%$ (squares), $10 \%$ (circles) and $20 \%$ (triangles).

The data are described satisfactorily by Eq. $3(a=0.5)$ at $c=10 \%$ and $20 \%$, while a slight deviation to a higher $a$ value is encountered for $c=5 \%$.

These results give evidence of the existence of two regimes within the 2-phase region: (1) Single-scattering regime, in the $\mathrm{T}$ range close to $\mathrm{T}_{\mathrm{PS}}\left(\mathrm{T}-\mathrm{T}_{\mathrm{PS}} \leq 4^{\circ} \mathrm{C}\right)$, where the singleexponential nature of $g_{2}(t)$ is preserved for at least $6 \mathrm{~h}$; (2) Multiple-scattering regime, in the $\mathrm{T}$ range well above $\mathrm{T}_{\mathrm{PS}}\left(\mathrm{T}-\mathrm{T}_{\mathrm{PS}} \geq 10^{\circ} \mathrm{C}\right)$, presumably by the formation of denser polymer aggregates. Confocal microscopy images taken at $c=5 \%$ in both regimes reveal that deeper $\mathrm{T}\left(\mathrm{T}-\mathrm{T}_{\mathrm{PS}} \geq 10^{\circ} \mathrm{C}\right)$ quenches lead to denser structures, as presented in Figure 7 , supporting the picture of multiple-scattering regime produced by denser particles.
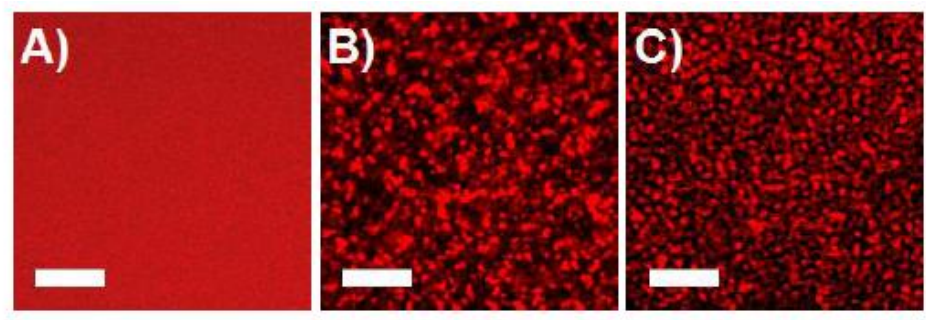

Figure 7. Confocal scanning microscopy images for $c=5 \%$ taken at (a) $30^{\circ} \mathrm{C}$ (clear solution), (b) $43^{\circ} \mathrm{C}$ and (c) $50^{\circ} \mathrm{C}$ after 30 min equilibration. The red features are attributed to water-rich domains, whereas the darker regions correspond to polymer-rich phases. Scale bar is $20 \mu \mathrm{m}$.

We now address the question of whether the characteristic size of polymer aggregates can be estimated in the single-scattering (DLS) and multiple-scattering (DWS) regimes. For comparison, the average distance (the domain length, $d_{\text {Confocal }}$ ) was determined by Fourier transform analysis of confocal microscopy images collected as time elapses at identical quench 
temperatures. The diffusion coefficient $(D)$ was obtained from the correlation time of the single mode $\left(\tau=1 / D q^{2}\right)$ above $\mathrm{T}_{\mathrm{PS}}$ (single-scattering regime) at different concentrations between 0.5 and $10 \%$. From $D$ values, we estimate the characteristic diameter $\left(d_{\text {DLS }}\right)$ of aggregates using the Stokes-Einstein relation, assuming spherical shapes of polymer domains and negligible interactions between them. The estimated aggregate sizes in the single-scattering regime after 30 min equilibration are in good agreement with $d_{\text {Confocal }}$ for dilute enough solutions $(c \leq 5 \%)$, as presented in Table 1 . However, the large differences observed for concentrated solutions ( $c=10 \%)$ manifest that the diffusion coefficient of the aggregates cannot be interpreted as originating from non-interacting objects and that the diffusion coefficient deviates from the dilute limit (Stokes-Einstein value).

Table 1. Characteristic HPC domain size in the two-phase region obtained in the concentration range $(0.5-10 \%)$.

\begin{tabular}{|c|c|c|c|c|}
\hline \multirow{2}{*}{$\boldsymbol{c}(\%)$} & \multicolumn{2}{|c|}{$\begin{array}{c}\text { Single-scattering regime } \\
\left(\mathrm{T}-\mathrm{T}_{\mathrm{PS}}=4^{\circ} \mathrm{C}\right)\end{array}$} & \multicolumn{2}{|c|}{$\begin{array}{c}\text { Multiple-scattering regime } \\
\left(\mathrm{T}-\mathrm{T}_{\mathrm{PS}}=10^{\circ} \mathrm{C}\right)\end{array}$} \\
\cline { 2 - 5 } & $d_{\mathrm{DLS}}(\mu \mathrm{m})$ & $d_{\text {Confocal }}(\mu \mathrm{m})$ & $d_{\mathrm{DWS}}(\mu \mathrm{m})$ & $d_{\text {Confocal }}(\mu \mathrm{m})$ \\
\hline 0.5 & $1.5 \pm 0.5$ & $3 \pm 1$ & $\left(^{*}\right)$ & $2 \pm 1$ \\
\hline 1 & $2 \pm 1$ & $4 \pm 1$ & $\left(^{*}\right)$ & $3 \pm 1$ \\
\hline 5 & $12 \pm 2$ & $10 \pm 2$ & $74 \pm 8(\gamma=1.33)$ & $4 \pm 1$ \\
& & & $3 \pm 1(\gamma=0.3)$ & \\
\hline 10 & $64 \pm 5$ & $9 \pm 2$ & $740 \pm 80(\gamma=1.33)$ & $3 \pm 1$ \\
& & & $3 \pm 1(\gamma=0.1)$ & \\
\hline
\end{tabular}

*Experimental data of $g_{2}(t)$ does not fit to Eq. 3

Regarding the multiple-scattering regime, fits of $g_{2}(t)$ to Eq. 3 assuming a $\gamma$ value of 1.33 (non-interacting particles) conducts to significant overestimations of the characteristic domain size with respect to confocal microscopy analysis, which evidence that the interactions between polymer aggregates cannot be neglected in the concentration range considered here. It is worth noting that Sanyal et al. ${ }^{39}$ showed that $\gamma$ decreases with the repulsive interactions between 
particles and found $\gamma$ values as low as 0.1 for strongly interacting systems. By fitting $g_{2}(t)$ to Eq. 3, assuming that $d_{\text {DWS }}=d_{\text {Confocal }}$ as obtained in the multiple-scattering regime, one gets a $\gamma$ value of the order of 0.3 at $c=5 \%$ and 0.1 at $c=10 \%$. Although this is consistent with expectations, a precise determination of $\gamma$ for HPC at different compositions is clearly out of the scope of this report.

Figure 8a shows the evolution of $d_{\mathrm{DLS}}$ with time at $c=5 \%$ (single-scattering regime, $43^{\circ} \mathrm{C}$ ). In the earlier stage of the experiment $(t \leq 10 \mathrm{~min}), d_{\mathrm{DLS}}$ satisfies a scaling law $d_{\mathrm{DLS}}(t) \sim \sqrt[3]{t}$, suggesting that HPC domain growth may follow a classical coarsening behavior. ${ }^{25,40}$ Note that for $t>10 \mathrm{~min}, d_{\mathrm{DLS}}$ reaches a plateau indicating that further coarsening of polymer domains is impeded. Such arrested phase separation behavior is consistent with the absence of macroscopic phase separation in the entire concentration range considered in this study (even after 5 days at $43^{\circ} \mathrm{C}$ ). DLS results compare favorably well with the growth kinetics captured by confocal microscopy (Figure 8).

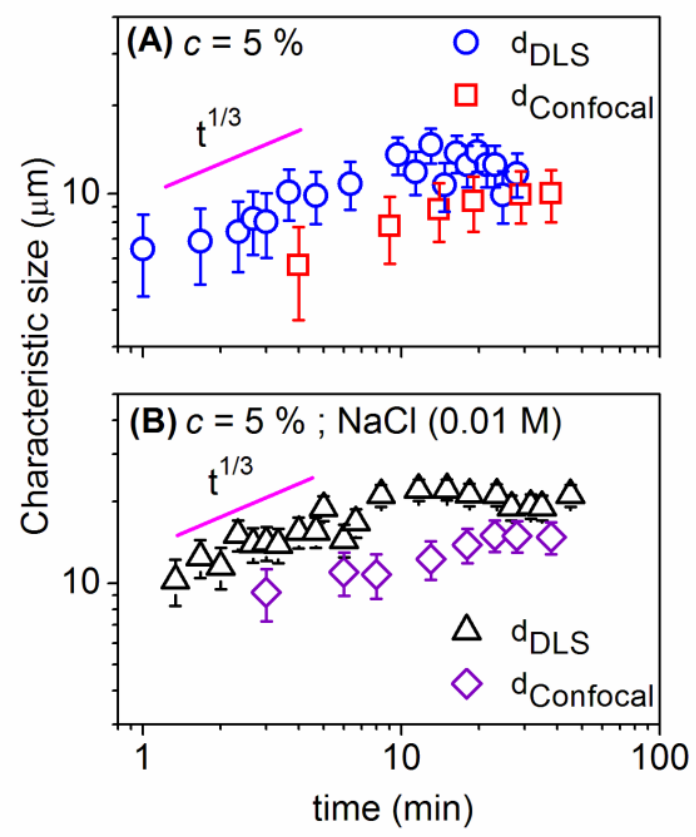

Figure 8. Evolution of characteristic dimension $d_{\mathrm{DLS}}$ and $d_{\text {Confocal }}$ as a function of time during phase separation of HPC solutions at $c=5 \%$ in (a) pure water and (b) $\mathrm{NaCl} 0.01 \mathrm{M}$. 
The arrested-like phase separation behavior observed for other LCST noninonic water-soluble polymers has been regarded as originating from gelation (methylcellulose, ${ }^{8,10}$ hydroxypropylmethylcellulose ${ }^{8}$ ) or from the effect of electrostatic charges at the interface of polymer aggregates inducing colloidal stability $\left(\right.$ PNIPAm $\left.^{31}\right)$. However, our results show that HPC phase separation is fundamentally different from those systems since phase separation does not induce gelation, and the arrested-like behavior after the growing stage persists by increasing the ionic strength (Figure 8b), which rules out a pure electrostatic effect as for the case of PNIPAm. In addition, confocal microscopy analysis revealed that the arrested phase separation behavior is also observed for higher $\mathrm{T}$ quenches $\left(50^{\circ} \mathrm{C}\right.$, data not shown) where the multiple-scattering regime is relevant. A potential explanation to the observed HPC arrestedlike phase separation mechanism could be the formation of polymer aggregates concentrated enough (glassy) to prevent colloidal coalescence. However, validation of this hypothesis would require direct measuring of the aggregate composition, and is left for a future work.

\section{Conclusions}

The thermo-responsive phase separation of commercial hydroxypropylcellulose was investigated in a broad concentration range covering the dilute and semi-dilute regime by dynamic light scattering. Our results show that the fast and slow mode amplitudes undergo a sharp transition by increasing the temperature near the phase separation temperature. Accordingly, we propose that by following those transitions, it is possible to define the phase separation boundary with a remarkable accuracy. Solutions with concentrations in the range $\left(c^{*}<c \leq c_{\mathrm{e}} \approx 10 c^{*}\right)$ undergo phase separation with a marked shift of $\tau_{\mathrm{sm}} T / \eta_{0}$ to higher values, reflecting clustering association at the phase separation condition. On the contrary, solutions in the range $c>c_{e}$ phase separate with a remarkable continuity of normalized 
relaxation times between the slow mode (below $\mathrm{T}_{\mathrm{PS}}$ ) and the single mode characteristic of the two-phase region. This behavior suggests that transient clusters formed in the single phase entangled region may act as precursors of polymer aggregates in the two-phase region, at temperatures close to $\mathrm{T}_{\mathrm{PS}}$.

The resulting phase separation diagram was compared to studies conducted by turbidimetric analysis using different criteria to define the phase boundary, showing that DLS transition temperatures reflect the onset of phase separation.

Within the two-phase region two temperature dependent regimes were identified. A singlescattering regime in the temperature range close to $\mathrm{T}_{\mathrm{PS}}\left(\mathrm{T}-\mathrm{T}_{\mathrm{PS}} \leq 4^{\circ} \mathrm{C}\right)$, characterized by slightly turbid samples. In this regime, monitoring growth kinetics of HPC solutions at $c \leq 5 \%$ it is possible by means of tracking the relaxation time of $g_{2}(t)$. Characteristic domain size growth at $c=5 \%$ follows the power law $d_{\mathrm{DLS}}(t) \sim \sqrt[3]{t}$ in the earlier stage of phase separation ( $t \leq 10 \mathrm{~min}$ ), suggesting a diffusive or coalescence/aggregation coarsening behavior. After the initial growing stage, the characteristic domain size levels off, suggesting an arrested-like phase separation mechanism, which inhibits macroscopic phase separation regardless of the ionic strength and quench temperature. A multiple-scattering regime was found at higher temperature quenches $\left(\mathrm{T}-\mathrm{T}_{\mathrm{PS}} \geq 10^{\circ} \mathrm{C}\right)$ at which the samples adopt a turbid and milky appearance. In this regime the system cannot be regarded as originating from non-interacting particles and therefore domain sizing and kinetic studies require a correct determination of $\gamma$ values for a DWS model to be applied in the back-scattering geometry.

We suggest that the method described here to map the phase separation diagram and kinetically resolve domain growth in the two-phase region is general and applies to other polymers displaying lower (or upper) critical solution temperature, provided the single scattering regime is correctly determined. 


\section{Acknowledgements}

This work was supported by the French National Research Agency, GASPOM Project, Grant $\mathrm{N}^{\mathrm{o}}$ ANR34GASPOMZ. H.G. acknowledges postdoctoral fellowship from CEA-Enhanced Eurotalents co-funded by FP7 Marie Skłodowska-Curie COFUND Programme, Grant agreement $\mathrm{N}^{\circ}$ 600382. The authors also thank Dr. Jan Ilavsky at Argonne National Laboratory for his help in using Clementine software and Prof. Catherine Amiel at Institut de Chimie et des Materiaux Paris-Est, CNRS for invaluable help in rheology experiments.

\section{References}

1. M. A. Cohen Stuart, W. T. S. Huck, J. Genzer, M. Müller, C. Ober, M. Stamm, G. B. Sukhorukov, I. Szleifer, V. V. Tsukruk, M. Urban, F. Winnik, S. Zausher, I. Luzinov and S. Minko, Emerging Applications of Stimuli-Responsive Polymer Materials, Nat. Mater., 2010, 9, 101-113.

2. O. M'Barki, A. Hanafia, D. Bouyer, C. Faur, R. Sescousse, U. Delabre, C. Blot, P. Guenoun, A. Deratani, D. Quemener and C. Pochat-Bohatier, Greener Method to Prepare Porous Polymer Membranes by Combining Thermally Induced Phase Separation and Crosslinking of Poly(vinyl alcohol) in Water, J. Membr. Sci., 2014, 458, 225-235.

3. A. Hanafia, C. Faur, A. Deratani, P. Guenoun, H. Garate, D. Quemener, C. Pochat-Bohatier and D. Bouyer, Fabrication of Novel Porous Membrane from Biobased Water-Soluble Polymer (Hydroxypropylcellulose), J. Membr. Sci., 2017, 526, 212-220.

4. A. Hanafia, D. Bouyer, C. Pochat Bohatier and C. Faur, Formation of Polymeric Porous Membrane without Organic Solvent by Thermally Induced Phase Separation in LCST System (Hydroxypropylcellulose/water), Procedia Eng., 2012, 44, 200-201.

5. A. Halperin, M. Kröger and F. M. Winnik, Poly( $N$-isopropylacrylamide) Phase Diagrams: Fifty Years of Research, Angew. Chem. Int. Ed., 2015, 54, 15342-15367.

6. S. Fortin and G. Charlet, Phase Diagram of Aqueous Solutions of (Hydroxypropyl)cellulose, Macromolecules, 1989, 22, 2286-2292.

7. E. Marsano and G. Fossati, Phase Diagram of Water Soluble Semirigid Polymers as a Function of Chain Hydrophobicity, Polymer, 2000, 41, 4357-4360.

8. J. P. A. Fairclough, H. Yu, O. Kelly, A. J. Ryan, R. L. Sammler and M. Radler, Interplay between Gelation and Phase Separation in Aqueous Solutions of Methylcellulose and Hydroxypropylmethylcellulose, Langmuir, 2012, 28, 10551-10557. 
9. M. Fettaka, R. Issaadi, N. Moulai-Mostefa, I. Dez, D. Le Cerf and L. Picton, Thermo Sensitive Behavior of Cellulose Derivatives in Dilute Aqueous Solutions: From Macroscopic to Mesoscopic Scale, J. Colloid Interface Sci., 2011, 357, 372-378.

10. S. A. Arvidson, J. R. Lott, J.W. McAllister, J. Zhang, F. S. Bates, T. P. Lodge, R. L. Sammler, Y. Li and M. Brackhagen, Interplay of Phase Separation and Thermoreversible Gelation in Aqueous Methylcellulose Solutions, Macromolecules, 2013, 46, 300-309.

11. J. Li, T. Ngai and C. Wu, The Slow Relaxation Mode: From Solutions to Gel Networks, Polymer Journal, 2010, 42, 609-625.

12. W. Brown and T. Nicolai, in Dynamic Light Scattering: The Method and Some Applications, ed. W. Brown, Clarendon Press, Oxford, 1993, 6, 272-318.

13. G. Yuan, X. Wang, C. C. Han, C. Wu, Reexamination of Slow Dynamics in Semi-Dilute Solutions: From Correlated Concentration Fluctuation to Collective Diffusion, Macromolecules, 2006, 39, 3642-3647.

14. J. F. Li, W. Li, H. Huo, S. Z. Luo and C. Wu. Reexamination of the Slow Mode in SemiDilute Polymer Solutions: The Effect of Solvent Quality, Macromolecules, 2008, 41, 901911.

15. W. Brown, K. Schillén, M. Almgren, S. Hvidt and P. Bahadur, Micelle and Gel Formation in a Poly(ethylene oxide)/Poly(propylene oxide)/Poly(ethylene oxide) Triblock Copolymer in Water Solution. Dynamic and Static Light Scattering and Oscillatory Shear Measurements, $J$. Phys. Chem., 1991, 95, 1850-1858.

16. W. Brown, M. Schillén and S. Hvidt, Triblock Copolymers in Aqueous Solution Studied by Static and Dynamic Light Scattering and Oscillatory Shear Measurements. Influence of Relative Block Sizes, J. Phys. Chem., 1992, 96, 6038-6044.

17. H. Chen, X. Ye, G. Zhang and Q. Zhang, Dynamics of Thermo-Responsive PNIPAM-gPEO Copolymer Chains in Semi-Dilute Solution, Polymer, 2006, 47, 8367-8373.

18. G. Fytas, H.G. Nothofer, U. Scherf, D. Vlassopoulos and G. Meier, Structure and Dynamics of Nondilute Polyfluorene Solutions, Macromolecules, 2002, 35, 481-488.

19. E. Somma, B. Loppinet, G. Fytas, S. Setayesh, J. Jacob, A.C. Grimsdale and K. Müllen, Collective Orientation Dynamics in Semi-rigid Polymers, Colloid Polym. Sci., 2004, 282, 867-873.

20. I. Yamamoto, K. Iwasaki and S. Hirotsu, Light Scattering Study of Condensation of Poly(N-isopropylacrylamide) Chain, J. Phys. Soc. Jpn., 1989, 58, 210-215.

21. G. Yuan, X. Wang, C. Han and C. Wu, Reexamination of Slow Dynamics in Semi-Dilute Solutions: Temperature and Salt Effects on Semi-Dilute Poly(N-isopropylacrylamide) Aqueous Solutions, Macromolecules, 2006, 39, 6207-6209.

22. J. Wang and C. Wu, Reexamination of the Origin of Slow Relaxation in Semi-Dilute Polymer Solutions-Reptation Related or Not?, Macromolecules, 2016, 49, 3184-3191. 
23. E. D. Klug, Some Properties of Water-soluble Hydroxyalkyl Celluloses and their Derivatives, J. Polym. Sci., Polym. Symp., 1971, 36, 491-508.

24. R. S. Werbowyj and D. G. Gray, Ordered Phase Formation in Concentrated Hydroxypropylcellulose Solutions, Macromolecules, 1980, 13, 69-73.

25. T. Kyu and P. Mukherjee, Kinetics of Phase Separation by Spinodal Decomposition in a Liquid-Crystalline Polymer Solution, Liq. Cryst., 1988, 3, 631-644.

26. R. H. Colby, Structure and Linear Viscoelasticity of Flexible Polymer Solutions: Comparison of Polyelectrolyte and Neutral Polymer Solutions, Rheol. Acta, 2010, 49, 425442.

27. B. Chu, Laser Light Scattering 2nd ed. Academic Press, New York, 1991.

28. Clementine software, http://www.igorexchange.com/project/clementine (accessed May 2017)

29. W. Brown and T. Nicolai, Static and Dynamic Behavior of Semidilute Polymer Solutions, Colloid Polym. Sci., 1990, 268, 977-990.

30. W. Brown and P. Štěpánek, Viscoelastic Relaxation in Semidilute and Concentrated Polymer Solutions, Macromolecules, 1993, 26, 6884-6890.

31. C. Balu, M. Delsanti and P. Guenoun, Colloidal Phase Separation of Concentrated PNIPAm Solutions, Langmuir, 2007, 23, 2404-2407.

32. H. Fujita, Polymer Solutions, Elsevier, Amsterdam, 1990.

33. K. Nishi, T. Hiroi, K. Hashimoto, K. Fujii, Y-S. Han, T-H. Kim, Y. Katsumoto and M. Shibayama, SANS and DLS Study of Tacticity Effects on Hydrophobicity and Phase Separation of Poly( $N$-isopropylacrylamide), Macromolecules, 2013, 46, 6225-6232.

34. B. Nystrom and R. Bergman, Velocity Sedimentation Transport Properties in Dilute and Concentrated Solutions of Hydroxypropyl Cellulose in Water at Different Temperatures up to Phase Separation, Eur. Polym. J., 1978, 14, 431-437.

35. A. Patra, P. Kumar Verma and R. Kumar Mitra, Slow Relaxation Dynamics of Water in Hydroxypropyl Cellulose-Water Mixture Traces Its Phase Transition Pathway: A Spectroscopic Investigation, J. Phys. Chem. B 2012, 116, 1508-1516.

36. G. Conio, E. Bianchi, A. Ciferri, A. Tealdi and M. A. Aden, Mesophase Formation and Chain Rigidity in Cellulose and Derivatives. 1. (Hydroxypropyl)cellulose in Dimethylacetamide, Macromolecules, 1983, 16, 1264-1270.

37. C. Lárez-V, V. Crescenzi and A. Ciferri, Phase Separation of Rigid Polymers in Poor Solvents.1. (Hydroxypropyl)cellulose in water, Macromolecules, 1995, 28, 5280-5284.

38. D. J. Pine, D. A. Weitz, J. X. Zhu and E. Herbolzheimer, Diffusing-wave Spectroscopy: Dynamic Light Scattering in the Multiple Scattering Limit, Journal de Physique, 1990, 51, 2101-2127. 
39. S. Sanyal and A. K. Sood, Diffusing Wave Spectroscopy of Dense Colloids: Liquid, Crystal and Glassy States, Pramana-J. Phys., 1995, 45, 1-17.

40. R. Kita, T. Kaku, K. Kubota and T. Dobashi, Pinning of Phase Separation of Aqueous Solution of Hydroxypropylmethylcellulose by Gelation, Phys. Lett. A, 1999, 259, 302-307. 